Short Note

\title{
Isolation and Characterization of 46 Novel Polymorphic EST-Simple Sequence Repeats (SSR) Markers in Two Sinipercine Fishes (Siniperca) and Cross-Species Amplification
}

\author{
Chunmei Qu, Xufang Liang *, Wei Huang and Liang Cao \\ College of Fisheries, Huazhong Agricultural University, Wuhan 430070, China; \\ E-Mails: linghanliuxiang@163.com (C.Q.); huangwei880924@163.com (W.H.); \\ caoliang0205@163.com (L.C.) \\ * Author to whom correspondence should be addressed; E-Mail: xufang_liang@hotmail.com; \\ Tel.: +86-27-8728-8255; Fax: +86-27-8728-2114.
}

Received: 9 July 2012; in revised form: 24 July 2012 / Accepted: 24 July 2012 /

Published: 30 July 2012

\begin{abstract}
With the development of next generation sequencing technologies, transcriptome level sequence collections are emerging as prominent resources for the discovery of gene-based molecular markers. In this study, we described the isolation and characterization of 46 novel polymorphic microsatellite loci for Siniperca chuatsi and Siniperca scherzeri from the transcriptome of their $\mathrm{F}_{1}$ interspecies hybrids. Forty-three of these loci were polymorphic in $S$. chuatsi, and 20 were polymorphic in $S$. scherzeri. In $S$. chuatsi, the number of alleles per locus ranged from 2 to 8 , and the observed and expected heterozygosities varied from 0.13 to 1.00 and from 0.33 to 0.85 , respectively. In $S$. scherzeri, the number of alleles per locus ranged from 3 to 9 , and the observed and expected heterozygosities varied from 0.19 to 1.00 and from 0.28 to 0.88 , respectively. We also evaluated the cross-amplification of 46 polymorphic loci in four species of sinipercine fishes: Siniperca kneri, Siniperca undulata, Siniperca obscura, and Coreoperca whiteheadi. The interspecies cross-amplification rate was very high, totaling $94 \%$ of the 184 locus/taxon combinations tested. These markers will be a valuable resource for population genetic studies in sinipercine fishes.
\end{abstract}

Keywords: Siniperca chuatsi; Siniperca scherzeri; EST-SSRs; transcriptome; cross-species amplification 


\section{Introduction}

Mandarin fish (Siniperca chuatsi), an economically important species in China, has a relatively high market value, and is wide cultured throughout the country [1,2]. It has a fast growth rate, but is susceptible to diseases. Compared with $S$. chuatsi, Golden mandarin fish (Siniperca scherzeri) has a great disease resistance, but grows slowly. Recently, outbreaks of diseases caused by parasites, bacteria and viruses have caused severe economic losses to the aquaculture industry [3]. In addition, because of overfishing, drought and especially water pollution, the wild stock of $S$. chuatsi is declining [4]. Therefore, breeding a disease-resistant and faster growing strain and preserving fish germplasm are becoming urgent aims in China.

Microsatellites or simple sequence repeats (SSRs) have become a useful tool to assess genetic diversity and develop molecular breeding techniques in fish due to their co-dominance, ubiquitous distribution within genomes, high reproducibility, and transferability across species [5,6]. However, the development of microsatellite markers has been limited by the labor and time required to construct, enrich, and sequence genomic libraries [7]. Fortunately, with the advent of next generation sequencing technologies, transcriptome sequencing is emerging as a rapid and efficient means for gene discovery and genetic marker development. Since EST-SSRs derived from transcriptome exist in the transcribed region of the genome, they can lead to the development of gene-based maps which help to identify candidate function genes and increase the efficiency of marker-assisted selection (MAS) [8]. Furthermore, EST-SSRs show a higher level of transferability to closely related species than non-EST-SSRs [9].

Although a few microsatellite markers were developed for $S$. chuatsi [10-14] and S. scherzeri [15], the number of available SSRs is grossly inadequate for genetic and mapping studies. Here, we describe the isolation and characterization of 46 novel polymorphic microsatellite loci for the S. chuatsi and S. scherzeri. We also test the transferability of these markers in other four species of sinipercine fishes: Siniperca kneri, Siniperca undulata, Siniperca obscura, and Coreoperca whiteheadi.

\section{Results and Discussion}

As shown in Table 1, a total of 46 polymorphic EST-SSR markers were newly developed. Forty-three of these loci were polymorphic in $S$. chuatsi, and 20 were polymorphic in S. scherzeri. Concerning $S$. chuatsi, the number of alleles per locus ranged from 2 to 8 , with an average of 4.3 alleles per locus. The observed $\left(H_{O}\right)$ and expected heterozygosities $\left(H_{E}\right)$ ranged from 0.13 to 1.00 (average of 0.55$)$ and from 0.33 to 0.85 (average of 0.63 ), respectively. In $S$. scherzeri, the number of alleles per locus ranged from 3 to 9 , with an average of 5.5 alleles per locus. The observed $\left(H_{O}\right)$ and expected heterozygosities $\left(H_{E}\right)$ ranged from 0.19 to 1.00 (average of 0.74 ) and from 0.28 to 0.88 (average of 0.72 ), respectively. Five loci (Sin134 in S. chuatsi, Sin118, Sin122, Sin158 and $\operatorname{Sin} 159$ in S. scherzeri) showed significant deviation from the Hardy-Weinberg equilibrium (HWE) after Bonferroni correction (adjusted $p$-value $=0.0012$ for $S$. chuatsi and 0.0026 for $S$. scherzeri), which may be due to the small sample size $(n=32)$ or the excess of heterozygotes. Another possible explanation for the departure from HWE is the dramatic contemporary decline in spawning populations, and consequent non-random mating and genetic bottlenecks [14]. No evidence for allelic dropout was found in these loci. 
No significant linkage disequilibrium (LD) was detected across all loci following Bonferroni correction (adjusted $p$-value $=0.0001$ for $S$. chuatsi and 0.0003 for $S$. scherzeri).

Overall, a high level of cross-species amplification was observed across the four species (Table 2). Forty-five of 46 polymorphic loci $(97.8 \%)$ were amplified successfully in $S$. undulate and $S$. obscura, $44(95.7 \%)$ in $S$. kneri, and $39(84.8 \%)$ in C. whiteheadi. These results were expected because of the taxonomical relationships of the families [16]. S. kneri, S. undulata, S. obscura are closely related to $S$. chuatsi and S. scherzeri, and all species belong to Siniperca, whereas $C$. whiteheadi is from Coreoperca which is sister genera to Siniperca. As transcriptome sequences are typically conserved relative to nontranscribed regions, SSRs residing in transcriptome sequences typically benefit from higher amplification rates and higher levels of cross-species transferability $[17,18]$. The high level of cross-species amplification tested here indicated not only the potential utility of transcriptome sequences for the identification and characterization of large numbers of gene-based SSR loci across species for which limited marker resources were available, but also the potential usefulness of the developed markers for a broader range of evolutionary, conservation and management studies in sinipercine fishes.

\section{Experimental Section}

De novo transcriptome sequencing of $\mathrm{F}_{1}$ hybrids between $S$. chuatsi (q) and S. scherzeri (đ) was performed and a total of 118,218 unigenes were identified. The processes of library preparation for transcriptome analysis and sequence assembly were as described in [19]. This unigene set was used for mining EST-SSR markers using the default parameters of the BatchPrimer3 v1.0 software [20]. In this study, a subset of 62 EST-SSR markers was screened on 32 S. chuatsi (Chibi, Hubei Province, China) and 32 S. scherzeri (Fengcheng, Liaoning Province, China), respectively. The primers for these SSR loci were designed using NCBI/Primer-BLAST [21].

Total genomic DNA was extracted from fin clips using the TIANamp Genomic DNA Kit (Tiangen) following the manufacturer's instructions. Polymerase chain reaction (PCR) conditions were optimized for each pair of primers. PCRs were performed in $25 \mu \mathrm{L}$ reaction volumes containing $2.5 \mu \mathrm{L}$ of $10 \times$ PCR buffer, 1.0-3.0 mM MgCl $2,50 \mu \mathrm{M}$ dNTPs, $0.4 \mu \mathrm{M}$ of each primer, $1 \mathrm{U}$ Taq polymerase (Takara) and $50 \mathrm{ng}$ genomic DNA. PCR conditions were as follows: initial denaturation at $94{ }^{\circ} \mathrm{C}$ for 3 min followed by 35 cycles at $94{ }^{\circ} \mathrm{C}$ for $30 \mathrm{~s}$, the optimized annealing temperature (Table 1) for $30 \mathrm{~s}, 72{ }^{\circ} \mathrm{C}$ for $30 \mathrm{~s}$, and then a final extension step at $72{ }^{\circ} \mathrm{C}$ for $10 \mathrm{~min}$. PCR products were separated on a $8 \%$ non-denaturing polyacrylamide gel electrophoresis and visualized by silver staining. A denatured pBR322 DNA/MspI molecular weight marker (Tiangen) was used as a size standard to identify alleles. 
Table 1. Characterization of 46 polymorphic EST-simple sequence repeats (SSR) markers in S. chuatsi and S. scherzeri.

\begin{tabular}{|c|c|c|c|c|c|c|c|c|c|c|}
\hline Locus & $\begin{array}{c}\text { Accession } \\
\text { number }\end{array}$ & Repeat motif & Primer sequence(5'-3') & $\begin{array}{c}\text { Size range } \\
\text { (bp) }\end{array}$ & $\begin{array}{c}T a \\
\left({ }^{\circ} \mathbf{C}\right)\end{array}$ & $\mathrm{Na}$ & $\boldsymbol{H}_{\boldsymbol{O}}$ & $\boldsymbol{H}_{E}$ & PIC & $p$-Value \\
\hline \multirow{2}{*}{$\operatorname{Sin} 109$} & \multirow{2}{*}{ JQ804765 } & \multirow{2}{*}{$(\mathrm{AG})_{15}$} & F: GGACACTGGACACTCAAACAT & $220-270$ & 54.5 & 4 & 0.2500 & 0.6339 & 0.5747 & 1.0000 \\
\hline & & & R: AGAGGATCAAAATTGTGCTTGAA & $246-285$ & 54.5 & 6 & 0.6875 & 0.8170 & 0.7756 & 0.9510 \\
\hline $\operatorname{Sin} 110$ & JQ804766 & $(\mathrm{AC})_{15}$ & R: AATCCAAGTGACAGGACGCC & - & - & - & - & - & - & - \\
\hline \multirow{2}{*}{$\operatorname{Sin} 112$} & \multirow{2}{*}{ JQ804768 } & \multirow{2}{*}{$(\mathrm{AC})_{15}$} & F: ATCGGCACCTGAGGCAAAAG & $132-166$ & 54.5 & 6 & 0.9688 & 0.7897 & 0.7444 & 0.0030 \\
\hline & & & R: GCCATCCATAGAGCCACGTC & 129-198 & 54.5 & 9 & 1.0000 & 0.8770 & 0.8479 & 0.0090 \\
\hline $\operatorname{Sin} 113$ & JQ804769 & $(\mathrm{TG})_{15}$ & R: GTGCACATGTCGAGTCAGTA & - & - & - & - & - & - & - \\
\hline \multirow{2}{*}{$\operatorname{Sin} 114$} & \multirow{2}{*}{ JQ804770 } & \multirow{2}{*}{$(\mathrm{AC})_{14}$} & F: AAGAGACAAGACACCACCGC & $185-209$ & 54.5 & 5 & 0.4375 & 0.6736 & 0.6012 & 0.9347 \\
\hline & & & R: ATGGTTTGACGGGAGACAGC & $194-243$ & 54.5 & 7 & 1.0000 & 0.8418 & 0.8064 & 0.0039 \\
\hline \multirow{2}{*}{$\operatorname{Sin} 116$} & \multirow{2}{*}{ JQ804771 } & \multirow{2}{*}{$(\mathrm{TG})_{14}(\mathrm{AG})_{7}$} & F: ACAATCCCAGCCCTCCTTCT & $212-265$ & 54.5 & 6 & 0.5625 & 0.8219 & 0.7813 & 0.9999 \\
\hline & & & R: GCAAGGTCCCTTTACATGCAG & $219-259$ & 54.5 & 5 & 0.8750 & 0.7877 & 0.7393 & 0.0798 \\
\hline $\operatorname{Sin} 117$ & JQ804772 & $(\mathrm{GT})_{14}$ & F: GGGCGGAAGACCAACTATGT & $268-291$ & 54.5 & 3 & 0.4062 & 0.5332 & 0.4697 & 0.9871 \\
\hline $\operatorname{Sin} 119$ & JQ804774 & $(\mathrm{CA})_{14}$ & R: ACCTCTGCTGCACAGCTAATC & - & - & - & - & - & - & - \\
\hline \multirow{2}{*}{$\operatorname{Sin} 120$} & \multirow{2}{*}{ JQ804775 } & \multirow{2}{*}{$(\mathrm{TTTG})_{7}$} & F: CCATCCCTCCGACCTTCAGT & $119-134$ & 54.5 & 4 & 0.5312 & 0.6900 & 0.6209 & 0.9418 \\
\hline & & & R: TTTAGGAACCCGACTCCGCT & - & - & - & - & - & - & - \\
\hline \multirow{2}{*}{$\operatorname{Sin} 122$} & & & F: TGCACTCACACACCTGTCTC & - & - & - & - & - & - & - \\
\hline & JQ804777 & $(\mathrm{TG})_{14} \mathrm{TAG}(\mathrm{GT})_{7}$ & R: AGCAGGATGCTTCATGCACTT & $205-246$ & 54.5 & 5 & 1.0000 & 0.6667 & 0.5927 & $0.0000 *$ \\
\hline & 1980 & & F: GATGGTGGTGAAACACTGGCT & $249-307$ & 54.5 & 6 & 0.7812 & 0.7773 & 0.7352 & 0.7478 \\
\hline $\operatorname{Sin} 123$ & JQ8047/8 & $(\mathrm{AC})_{14}$ & R: GTGTTGAGAGGGTCCTGGTG & $198-214$ & 56.0 & 3 & 0.5000 & 0.5397 & 0.4683 & 0.9912 \\
\hline
\end{tabular}


Table 1. Cont.

\begin{tabular}{|c|c|c|c|c|c|c|c|c|c|c|}
\hline Locus & $\begin{array}{c}\text { Accession } \\
\text { number }\end{array}$ & Repeat motif & Primer sequence(5'-3') & $\begin{array}{c}\text { Size range } \\
\text { (bp) }\end{array}$ & $\begin{array}{c}\mathrm{Ta} \\
\left({ }^{\circ} \mathrm{C}\right) \\
\end{array}$ & $N a$ & $H_{o}$ & $\boldsymbol{H}_{E}$ & $P I C$ & $p$-Value \\
\hline \multirow{2}{*}{$\operatorname{Sin} 124$} & \multirow{2}{*}{ JQ804779 } & \multirow{2}{*}{$(\mathrm{CA})_{14}$} & F: TCAAACACCACCCACCCCTG & $248-281$ & 54.5 & 4 & 0.8750 & 0.7297 & 0.6667 & 0.0180 \\
\hline & & & R: ACCGGGACAGGATGGGAGTC & - & - & - & - & - & - & - \\
\hline \multirow{2}{*}{$\operatorname{Sin} 125$} & \multirow{2}{*}{ JQ804780 } & \multirow{2}{*}{$(\mathrm{CA})_{14}$} & F: ACCCTCTGTGTGGCGAATGT & $277-311$ & 54.5 & 3 & 0.6250 & 0.6265 & 0.5474 & 0.5368 \\
\hline & & & R: CGGGACAGGATGGGAGTCG & - & - & - & - & - & - & - \\
\hline \multirow{2}{*}{$\operatorname{Sin} 127$} & \multirow{2}{*}{ JQ804782 } & \multirow{2}{*}{$(\mathrm{TG})_{14}$} & F: AGACGTAGCCCAGGCTCAAA & $215-251$ & 54.5 & 3 & 0.5938 & 0.5055 & 0.4213 & 0.0303 \\
\hline & & & R: TGTGGGGTTCACTACAGGGT & - & - & - & - & - & - & - \\
\hline \multirow{2}{*}{$\operatorname{Sin} 128$} & \multirow{2}{*}{ JQ804783 } & \multirow{2}{*}{$(\mathrm{AC})_{14}$} & F: CTGTGCCTCAGTGTGCTGC & $225-257$ & 54.5 & 3 & 0.4062 & 0.6394 & 0.5572 & 0.9995 \\
\hline & & & R: ACTTGTAATGGGCAAATTGTCACT & - & - & - & - & - & - & - \\
\hline \multirow{2}{*}{$\operatorname{Sin} 129$} & \multirow{2}{*}{ JQ804784 } & \multirow{2}{*}{$(\mathrm{CA})_{14}$} & F: ACGCTGCGAGGTGTGATATG & $131-164$ & 54.5 & 5 & 0.6250 & 0.7500 & 0.6971 & 0.9857 \\
\hline & & & R: CTGGCCCTCGTTAGTGCTTG & $185-217$ & 54.5 & 8 & 1.0000 & 0.8457 & 0.8103 & 0.0046 \\
\hline \multirow{2}{*}{$\operatorname{Sin} 130$} & \multirow{2}{*}{ JQ804785 } & \multirow{2}{*}{$\left(\mathrm{GTGA}_{7} \mathrm{~N}_{7}(\mathrm{TG})_{8}\right.$} & F: CTCGCAGGCTTTTCTCTGCT & $282-300$ & 54.5 & 2 & 0.3438 & 0.3963 & 0.3140 & 0.8902 \\
\hline & & & R: AGCCATCAGTTCTGTTCTTTCTT & $252-280$ & 54.5 & 5 & 0.7188 & 0.7485 & 0.6982 & 0.3062 \\
\hline \multirow{2}{*}{$\operatorname{Sin} 131$} & \multirow{2}{*}{ JQ804785 } & \multirow{2}{*}{$(\mathrm{ATGG})_{7}$} & F: GGAGGAAAATAATTTCATTTGGGAT & $180-200$ & 54.5 & 3 & 0.1250 & 0.4107 & 0.3665 & 0.9998 \\
\hline & & & R: GTCATTGCATTCAAAAGTTAGGCT & - & - & - & - & - & - & - \\
\hline \multirow{2}{*}{$\operatorname{Sin} 134$} & \multirow{2}{*}{ JQ804789 } & \multirow{2}{*}{$(\mathrm{TG})_{14}$} & F: GCCCCCTTCTCAACCCACTA & $106-120$ & 54.5 & 6 & 1.0000 & 0.8075 & 0.7645 & $0.0008 *$ \\
\hline & & & R: TGCTTTCCAAAGCGAACCGT & $108-134$ & 54.5 & 8 & 0.9688 & 0.8621 & 0.8299 & 0.0451 \\
\hline \multirow{2}{*}{$\operatorname{Sin} 135$} & \multirow{2}{*}{ JQ804790 } & \multirow{2}{*}{$(\mathrm{TG})_{14}$} & F: GTGATATCTCCTCCTGACGGC & $273-302$ & 54.5 & 4 & 0.5938 & 0.5585 & 0.4900 & 0.3484 \\
\hline & & & R: ACATTCTGAATTGCAAAGGCTCA & - & - & - & - & - & - & - \\
\hline \multirow{2}{*}{$\operatorname{Sin} 136$} & \multirow{2}{*}{ JQ804791 } & \multirow{2}{*}{$(\mathrm{TG})_{14}$} & F: AACTGAAATGTGTGGTGAACTGA & $138-164$ & 56.8 & 5 & 0.5000 & 0.7202 & 0.6585 & 0.9243 \\
\hline & & & R: GTGTCTCCCAACAAGTGGCA & - & - & - & - & - & - & - \\
\hline \multirow{2}{*}{$\operatorname{Sin} 137$} & IO80479? & (TCA) & F: AGCGTCTACTGAGGGTCAAACT & $234-280$ & 54.5 & 5 & 0.4688 & 0.7207 & 0.6700 & 0.9960 \\
\hline & गरण 192 & $(1<A) 9$ & R: GGTGGACTGACCAGCAAGGA & - & - & - & - & - & - & - \\
\hline Sin120 & 10801702 & $(\Delta T C)$ & F: TCATCTGAGGACGACTCGCT & $229-253$ & 52.5 & 3 & 0.4375 & 0.5997 & 0.5025 & 0.9772 \\
\hline $\sin 138$ & JQ804/93 & $(\mathrm{A} I \mathrm{C})_{9}$ & R: AACTTAACTTCCTGCTGTCCCT & - & - & - & - & - & - & - \\
\hline
\end{tabular}


Table 1. Cont.

\begin{tabular}{|c|c|c|c|c|c|c|c|c|c|c|}
\hline Locus & $\begin{array}{c}\text { Accession } \\
\text { number }\end{array}$ & Repeat motif & Primer sequence(5'-3') & $\begin{array}{c}\text { Size range } \\
\text { (bp) }\end{array}$ & $\begin{array}{c}\mathrm{Ta} \\
\left({ }^{\circ} \mathrm{C}\right)\end{array}$ & $N a$ & $\boldsymbol{H}_{\boldsymbol{O}}$ & $\boldsymbol{H}_{E}$ & PIC & $p$-Valuc \\
\hline \multirow{2}{*}{ Sin 139} & \multirow{2}{*}{ JQ804794 } & \multirow{2}{*}{$(\mathrm{CTC})_{9}$} & F: GTGACTGCATCCAGGTGTCG & - & - & - & - & - & - & - \\
\hline & & & R: GGCCGAGGTCGGTTGTTATC & $189-207$ & 54.5 & 3 & 0.1875 & 0.2803 & 0.2584 & 0.9947 \\
\hline \multirow{2}{*}{$\operatorname{Sin} 140$} & \multirow{2}{*}{ JQ804795 } & \multirow{2}{*}{$(\mathrm{TCA})_{9}$} & F: TGTGGTTCTCCTCTCCCACA & $253-304$ & 53.2 & 5 & 0.4375 & 0.7336 & 0.6759 & 0.9985 \\
\hline & & & R: AGAGGTTGGTGCAGGAGACTT & - & - & - & - & - & - & - \\
\hline \multirow{2}{*}{$\operatorname{Sin} 142$} & \multirow{2}{*}{ JQ804797 } & \multirow{2}{*}{$(\mathrm{CTT})_{9}$} & F: CATCAACGCAATGCAAGGGT & $150-180$ & 54.5 & 2 & 0.1562 & 0.3289 & 0.2713 & 0.9997 \\
\hline & & & R: CTGGAGCCGGACTTGAGGAA & $181-226$ & 54.5 & 6 & 0.3438 & 0.6994 & 0.6492 & 0.9998 \\
\hline \multirow{2}{*}{$\operatorname{Sin} 143$} & \multirow{2}{*}{ JQ804797 } & \multirow{2}{*}{$(\mathrm{GTT})_{7}$} & F: AAAGCAGGCCAAACAACACC & $198-246$ & 54.5 & 5 & 0.4062 & 0.7733 & 0.7206 & 1.0000 \\
\hline & & & R: AGGACGGGGAGGCTTTTGAT & - & - & - & - & - & - & - \\
\hline \multirow{2}{*}{$\operatorname{Sin} 146$} & \multirow{2}{*}{ JQ804800 } & \multirow{2}{*}{$\begin{array}{c}(\mathrm{GAG})_{6} \mathrm{~N}_{5} \\
(\mathrm{AAG})_{9}\end{array}$} & F: GTAATCGACACGGACAGCGA & $370-452$ & 54.5 & 4 & 0.5938 & 0.6781 & 0.6069 & 0.8240 \\
\hline & & & R: CACACACATTCTCCTCAGCGT & - & - & - & - & - & - & - \\
\hline \multirow{2}{*}{$\operatorname{Sin} 147$} & \multirow{2}{*}{ JQ804801 } & \multirow{2}{*}{$(\mathrm{TCC})_{9}$} & F: AGATCAGACACCAGGAGGACC & $174-232$ & 53.5 & 5 & 0.3125 & 0.7242 & 0.6576 & 0.9971 \\
\hline & & & R: AAGACGGAGGCAAAGAACGAC & $192-225$ & 54.5 & 4 & 0.5625 & 0.7614 & 0.7026 & 0.9964 \\
\hline \multirow{2}{*}{$\operatorname{Sin} 148$} & \multirow{2}{*}{ JQ804802 } & \multirow{2}{*}{$(\mathrm{CAT})_{9}$} & F: CGAGGCCAGGAGTGAACCAA & $255-303$ & 53.5 & 3 & 0.6875 & 0.4866 & 0.4009 & 0.0027 \\
\hline & & & R: GCACAGCTGGAGGTGTTTCG & - & - & - & - & - & - & - \\
\hline \multirow{2}{*}{$\operatorname{Sin} 151$} & \multirow{2}{*}{ JQ804805 } & \multirow{2}{*}{$(\mathrm{GT})_{13}$} & F: GTGCAAGGCCTTAGTCTCTCC & $170-221$ & 55.5 & 4 & 0.6875 & 0.6434 & 0.5761 & 0.8897 \\
\hline & & & R: GCCCACCAGATCTACCGAGT & - & - & - & - & - & - & - \\
\hline \multirow{2}{*}{$\operatorname{Sin} 152$} & \multirow{2}{*}{ JQ804806 } & \multirow{2}{*}{$(\mathrm{AG})_{8} \mathrm{~A}(\mathrm{AG})_{13}$} & F: TGCGCCACTTTACTGATGGG & $173-242$ & 53.5 & 6 & 0.6562 & 0.8105 & 0.7677 & 0.9164 \\
\hline & & & R: GCATTAACCAAACCCCGCGA & $185-240$ & 54.5 & 8 & 0.9688 & 0.8522 & 0.8193 & 0.1740 \\
\hline \multirow{2}{*}{$\operatorname{Sin} 153$} & \multirow{2}{*}{ JQ804807 } & \multirow{2}{*}{$(\mathrm{AG})_{13}$} & F: GCACAGGTTTTTCTAAACATTGCT & $155-208$ & 53.5 & 5 & 0.1875 & 0.3795 & 0.3538 & 0.9662 \\
\hline & & & R: TGTTGTTATTGTCAGTGTGTTTTCT & $177-215$ & 54.5 & 4 & 0.2500 & 0.6806 & 0.6160 & 1.0000 \\
\hline \multirow{2}{*}{$\operatorname{Sin} 154$} & I0804808 & & F: ACTGGTTTGTGGTTTGGAGGT & $211-229$ & 53.5 & 2 & 0.5625 & 0.4107 & 0.3225 & 0.0356 \\
\hline & JQ804808 & $(\mathrm{G} 1)_{13}$ & R: ATGATTTTTCTTGCCTTCGTGT & - & - & - & - & - & - & - \\
\hline $\operatorname{Sin} 155$ & IO804809 & $(A C)$ & F: GAATGGTGTGTTGCACAGCG & $157-190$ & 53.5 & 3 & 0.2188 & 0.3894 & 0.3473 & 0.9939 \\
\hline $\sin 155$ & JQ804809 & $(A C)_{13}$ & R: CATTCTAGCATGTGCGAGGC & $160-201$ & 54.5 & 7 & 0.6875 & 0.8021 & 0.7600 & 0.9004 \\
\hline
\end{tabular}


Table 1. Cont

\begin{tabular}{|c|c|c|c|c|c|c|c|c|c|c|}
\hline Locus & $\begin{array}{c}\text { Accession } \\
\text { number }\end{array}$ & Repeat motif & Primer sequence(5'-3') & $\begin{array}{c}\text { Size range } \\
\text { (bp) }\end{array}$ & $\begin{array}{c}T a \\
\left({ }^{\circ} \mathrm{C}\right) \\
\end{array}$ & $N a$ & $\boldsymbol{H}_{\boldsymbol{O}}$ & $\boldsymbol{H}_{E}$ & PIC & $p$-Value \\
\hline \multirow{2}{*}{$\operatorname{Sin} 156$} & \multirow{2}{*}{ JQ804810 } & \multirow{2}{*}{$(\mathrm{AC})_{13}$} & F: TAGGAGGCTTTACAACCGGC & $188-205$ & 53.5 & 2 & 0.5625 & 0.4107 & 0.3225 & 0.0344 \\
\hline & & & R: ATGACCAGCCTCAGGTGTCT & - & - & - & - & - & - & - \\
\hline \multirow{2}{*}{$\operatorname{Sin} 157$} & \multirow{2}{*}{ JQ804811 } & \multirow{2}{*}{$(\mathrm{AC})_{13}$} & F: CATTTGCTGGCTCTCACACC & $184-215$ & 53.5 & 3 & 0.5000 & 0.4330 & 0.3477 & 0.2070 \\
\hline & & & R: TGTTTAATTCATGCCTAGGTTTAGT & - & - & - & - & - & - & - \\
\hline \multirow{2}{*}{$\operatorname{Sin} 158$} & \multirow{2}{*}{ JQ804812 } & \multirow{2}{*}{$(\mathrm{CA})_{13}$} & F: TGAGAACTGCCTGAGCCGAG & - & - & - & - & - & - & - \\
\hline & & & R: CTGCAGAGCCGTGGAGACTA & $210-248$ & 54.5 & 3 & 0.9062 & 0.5303 & 0.4145 & $0.0000 *$ \\
\hline \multirow{2}{*}{$\operatorname{Sin} 159$} & \multirow{2}{*}{ JQ804813 } & \multirow{2}{*}{$(\mathrm{TG})_{13}$} & F: CGCTGATCGCTCTGTGCTCCC & $196-234$ & 53.5 & 5 & 0.6875 & 0.7614 & 0.7108 & 0.7259 \\
\hline & & & R: ACACGGAAGCTGGTGAGCGG & $199-233$ & 56.0 & 5 & 0.9688 & 0.6379 & 0.5682 & $0.0000 *$ \\
\hline \multirow{2}{*}{$\operatorname{Sin} 160$} & \multirow{2}{*}{ JQ804814 } & \multirow{2}{*}{$(\mathrm{TG})_{13}$} & F: CCACTGGAGCCCACATGGCA & $307-360$ & 55.5 & 5 & 0.8125 & 0.6711 & 0.6123 & 0.0151 \\
\hline & & & R: TGAGTGGGCGCTACTGTGTGT & $291-331$ & 54.5 & 5 & 0.7812 & 0.7659 & 0.7137 & 0.5928 \\
\hline \multirow{2}{*}{$\operatorname{Sin} 162$} & \multirow{2}{*}{ JQ804815 } & \multirow{2}{*}{$(\mathrm{TA})_{13}$} & F: TGCTTTGCTGGTTGGCAGGCT & $294-368$ & 53.5 & 5 & 0.1875 & 0.6270 & 0.5651 & 1.0000 \\
\hline & & & R: CGTGGAGGTGCGACGCGTAA & - & - & - & - & - & - & - \\
\hline \multirow{2}{*}{$\operatorname{Sin} 163$} & \multirow{2}{*}{ JQ804817 } & \multirow{2}{*}{$(\mathrm{CA})_{13}$} & F: ACAGCCAGGCTCCTCCACCT & $230-269$ & 53.5 & 8 & 0.6875 & 0.8482 & 0.8134 & 0.9597 \\
\hline & & & R: TCTTTCACAGGCAAACCACTGCT & $225-273$ & 53.5 & 6 & 0.4688 & 0.7803 & 0.7392 & 1.0000 \\
\hline \multirow{2}{*}{$\operatorname{Sin} 166$} & \multirow{2}{*}{ JQ804819 } & \multirow{2}{*}{$(\mathrm{GA})_{13}$} & F: GAAATTGAAGAAGACAAGGTGATG & $204-231$ & 53.5 & 3 & 0.2500 & 0.4504 & 0.4012 & 0.9998 \\
\hline & & & R: CTGCTTTTGGCAGGAGCTAA & - & - & - & - & - & - & - \\
\hline \multirow{2}{*}{$\operatorname{Sin} 169$} & \multirow{2}{*}{ JQ804822 } & \multirow{2}{*}{$(\mathrm{AC})_{13}$} & F: TGACAAATCACTGGGTTTACTCCT & $214-284$ & 53.5 & 5 & 0.5625 & 0.6443 & 0.5790 & 0.9164 \\
\hline & & & R: GACATGCTGCTCTCCGATCC & - & - & - & - & - & - & - \\
\hline \multirow{2}{*}{$\operatorname{Sin} 170$} & \multirow{2}{*}{ JQ804823 } & \multirow{2}{*}{$(\mathrm{GT})_{13}$} & F: CTTGAGTGGTTGATTGTGCCCT & $242-270$ & 55.5 & 4 & 0.7188 & 0.5491 & 0.4990 & 0.0015 \\
\hline & & & R: GCAGACATTGCTGAGGGATGAA & - & - & - & - & - & - & - \\
\hline
\end{tabular}

For each locus the information in the top row refers to $S$. chuatsi and the second row refers to $S$. scherzeri. Ta corresponds to annealing temperature; $N a$ is number of alleles; $H_{O}$ and $H_{E}$ are observed and expected heterozygosity, respectively; PIC is the polymorphic information content. * indicates significant deviation from HWE after Bonferroni correction; no polymorphism for each locus is denoted by "_-”. 
The number of alleles $(\mathrm{Na})$, the observed $\left(\mathrm{H}_{O}\right)$ and expected heterozygosities $\left(H_{E}\right)$ were estimated using POPGENE version 1.32 [22]. The polymorphic information content (PIC) was calculated using the formula:

$$
P I C=1-\left(\sum_{i=1}^{n} q_{i}^{2}\right)-\left(\sum_{i=1}^{n-1} \sum_{j=i+1}^{n} 2 q_{i}^{2} q_{j}^{2}\right)
$$

where $n$ is the number of alleles, and $q_{i}, q_{j}$ is the $i$ th and $j$ th allele frequency, respectively [23]. Deviations from Hardy-Weinberg equilibrium (HWE) and linkage disequilibrium (LD) were tested using the online version of GENEPOP [24]. All results were adjusted for multiple simultaneous comparisons using a sequential Bonferroni correction [25]. Genotyping errors due to null alleles, stutter bands, or allele dropout were analyzed using the software Micro-checker 2.2.3 [26].

Cross-species amplification of the above-developed polymorphic SSR loci was tested in four species of sinipercine fishes: S. kneri, S. undulata, S. obscura, and C. whiteheadi. Two individuals of each species were analyzed. The same PCR conditions were used as described above except that the annealing temperature was re-optimized at each locus (Table 2). Amplification products were visualized in $1.5 \%$ agarose gels, and fragments were sized by comparison with a $2 \mathrm{~kb}$ DNA Marker (Trans). Primer pairs that amplified fragments with similar sizes to those observed in source species were considered as successful cross-species amplification.

Table 2. Cross-species amplification for the 46 polymorphic EST-SSR markers in four species of sinipercine fishes.

\begin{tabular}{lcccc}
\hline & \multicolumn{4}{c}{ Species } \\
\cline { 2 - 5 } Locus & S. undulate & S. obscura & S. kneri & C. whiteheadi \\
\hline Sin109 & 54.5 & 54.5 & 54.5 & 54.5 \\
Sin110 & 54.5 & 54.5 & 54.5 & 54.5 \\
Sin112 & 54.5 & 54.5 & 54.5 & 54.5 \\
Sin113 & 54.5 & 54.5 & 54.5 & 54.5 \\
Sin114 & 54.5 & 54.5 & 54.5 & 54.5 \\
Sin116 & 54.5 & 54.5 & 54.5 & - \\
Sin117 & 54.5 & 54.5 & 54.5 & - \\
Sin118 & 54.5 & 54.5 & 54.5 & 54.5 \\
Sin119 & 54.5 & 54.5 & 54.5 & 54.5 \\
Sin120 & 54.5 & 54.5 & 54.5 & 54.5 \\
Sin122 & 54.5 & 54.5 & 54.5 & 54.5 \\
Sin123 & 54.5 & 54.5 & 54.5 & 54.5 \\
Sin124 & 54.5 & 54.5 & 54.5 & 54.5 \\
Sin125 & 54.5 & 54.5 & 54.5 & 54.5 \\
Sin127 & 54.5 & 54.5 & 54.5 & 54.5 \\
Sin128 & 54.5 & 54.5 & 54.5 & - \\
Sin129 & 54.5 & 54.5 & -5 & 54.5 \\
Sin130 & 54.5 & 54.5 & 54.5 & 54.5 \\
Sin131 & 54.5 & 54.5 & 54.5 & 54.5 \\
Sin134 & 54.5 & 54.5 & 54.5 & 54.5 \\
Sin135 & 54.5 & 54.5 & 54.5 & - \\
Sin136 & 54.5 & 54.5 & 54.5 & 54.5 \\
Sin137 & 54.5 & 54.5 & 54.5 & \\
\hline & & & & 54.5 \\
\hline
\end{tabular}


Table 2. Cont.

\begin{tabular}{lcccc}
\hline & \multicolumn{3}{c}{ Species } \\
\cline { 2 - 5 } Locus & S. undulate & S. obscura & S. kneri & C. whiteheadi \\
\hline Sin138 & 54.5 & 54.5 & 54.5 & 54.5 \\
Sin139 & 54.5 & 54.5 & 54.5 & 54.5 \\
Sin140 & 54.5 & 54.5 & 54.5 & 54.5 \\
Sin142 & 54.5 & 54.5 & 54.5 & 54.5 \\
Sin143 & 54.5 & 54.5 & 54.5 & 54.5 \\
Sin146 & 54.5 & 54.5 & 54.5 & - \\
Sin147 & 54.5 & 54.5 & 54.5 & 54.5 \\
Sin148 & 54.5 & 54.5 & 54.5 & 54.5 \\
Sin151 & 54.5 & 54.5 & 54.5 & 54.5 \\
Sin152 & 54.5 & 54.5 & 54.5 & 54.5 \\
Sin153 & 54.5 & 54.5 & 54.5 & 54.5 \\
Sin154 & 54.5 & 54.5 & 54.5 & 54.5 \\
Sin155 & - & - & -5 & - \\
Sin156 & 54.5 & 54.5 & 54.5 & 54.5 \\
Sin157 & 54.5 & 54.5 & 54.5 & 54.5 \\
Sin158 & 54.5 & 54.5 & 54.5 & 54.5 \\
Sin159 & 54.5 & 54.5 & 54.5 & 52.8 \\
Sin160 & 54.5 & 54.5 & 54.5 & - \\
Sin162 & 54.5 & 54.5 & 57.0 & 51.1 \\
Sin163 & 54.5 & 54.5 & 54.5 & 52.8 \\
Sin166 & 54.5 & 54.5 & 54.5 & 54.5 \\
Sin169 & 54.5 & 54.5 & 54.5 & 54.5 \\
Sin170 & 54.5 & 54.5 & 54.5 & \\
\hline
\end{tabular}

The annealing temperature for each locus was shown. Unsuccessful amplification of PCR products for each locus is denoted by "-_".

\section{Conclusions}

In summary, a total of 46 polymorphic EST-SSR markers were newly developed. Forty-three of these loci were polymorphic in $S$. chuatsi, and 20 were polymorphic in $S$. scherzeri. We only tested a small subset of the SSR loci identified in our transcriptome, but high levels of polymorphism, and high level of cross-species amplification indicate that the pairs of primers described here may be suitable for assessments of genetic diversity and population structure, the construction of high-density linkage map, conservation and molecular marker-assisted breeding in many species of sinipercine fishes. Our results highlight the value of next generation transcriptome resources for the characterization and development of gene-based SSRs.

\section{Acknowledgments}

This work was financially supported by the National Natural Science Foundation of China (31172420), the National Basic Research Program of China (2009CB118702) and the Fundamental Research Funds for the Central Universities (2010PY010, 2011PY030). 


\section{References}

1. Liang, Y.; Cui, X. The eco-physiological characteristics of artificial propagation in mandarin fish (Siniperca chuatsi). Acta Hydrobiol. Sin. 1982, 16, 90-92.

2. Liu, J.; Cui, Y.; Liu, J. Food consumption and growth of two piscivorous fishes, the mandarin fish and the Chinese snakehead. J. Fish Biol. 1998, 53, 1071-1083.

3. He, J.G.; Zeng, K.; Weng, S.P.; Chan, S.M. Experimental transmission, pathogenicity and physical-chemical properties of infectious spleen and kidney necrosis virus (ISKNV). Aquaculture 2002, 204, 11-24.

4. Zhang, C.; Zhao, Y. The resources status, recovery and reasonable utilization of Siniperca chuatsi in China (in Chinese). Bull. Biol. 1999, 34, 9-11.

5. Walter, R.; Epperson, B.K. Geographic pattern of genetic variation in Pinus resinosa: Area of greatest diversity is not the origin of postglacial populations. Mol. Ecol. 2001, 10, 103-111.

6. Saha, M.C.; Cooper, J.D.; Rouf Mian, M.A.; Chekhovskiy, K.; May, G.D. Tall fescue genomic SSR markers: Development and transferability across multiple grass species. Theor. Appl. Genet. 2006, 113, 1449-1458.

7. Edwards, K.J.; Barker, J.H.; Daly, A.; Jones, C.; Karp, A. Microsatellite libraries enriched for several microsatellite sequences in plants. BioTechniques 1996, 20, 758-760.

8. Gupta, P.K.; Rustgi, S. Molecular markers from the transcribed/expressed region of the genome in higher plants. Funct. Integr. Genomics 2004, 4, 139-162.

9. Saha, M.C.; Mian, M.A.; Eujay, I.; Zwonitzer, J.C.; Wang, L.; May, G.D. Tall fescue EST-SSR markers with transfer ability across several grass species. Theor. Appl. Genet. 2004, 109, 783-791.

10. Zhang, B.; Li, Z.J.; Tong, J.G.; Liao, X.L. Isolation and characterization of 18 polymorphic microsatellite markers in Chinese mandarin fish Siniperca chuatsi (Basilewsky). Mol. Ecol. Notes 2006, 6, 1216-1218.

11. Kuang, G.Q.; Liu, Z.; Lu, S.Q.; Liu, H.Y.; Zhang, J.S.; Tang, J.Z. Isolation and characterization of microsatellite loci from Siniperca chuatsi (in Chinese). J. Fish Sci. China 2007, 14, 608-614.

12. Kuang, G.Q.; Liu, Z.; Lu, S.Q.; Liu, H.Y.; Zhang, J.S.; Xiao, T.Y. Isolation and characterization of microsatellite loci of Siniperca chuatsi from GenBank database (in Chinese). Acta Zool. Sin. 2007, 53, 184-189.

13. Kuang, G.Q.; Lu, S.Q.; Zheng, S.M.; Wu, Q. Isolation and evaluation of 18 microsatellite loci in Siniperca chuatsi (Basilewsky). Mol. Ecol. Resour. 2009, 9, 1473-1475.

14. Liu, X.L.; Luo, W.; Zeng, C.; Wang, W.M.; Gao, Z.X. Isolation of New 40 Microsatellite Markers in Mandarin Fish (Siniperca chuatsi). Int. J. Mol. Sci. 2011, 12, 4180-4189.

15. Yang, M.; Liang, X.F.; Tian, C.X.; Gul, Y.; Dou, Y.Q.; Cao, L.; Yu, R. Isolation and characterization of fifteen novel microsatellite loci in golden mandarin fish (Siniperca scherzeri) Steindachne. Conserv. Genet. Resour. 2012, doi: 10.1007/s12686-012-9601-1.

16. Liu, H.Z. Studies on Skeleton Anatomy and Phylogeny of the Sinipericine Fishes. Ph.D. Dissertation, Institute of Hydrobiology, Chinese Academy of Sciences, Wuhan, Hubei Province, 1993.

17. Barbara, T.; Palma-Silva, C.; Paggi, G.M.; Bered, F.; Fay, M.F.; Lexer, C. Cross-species transfer of nuclear microsatellite markers: potential and limitations. Mol. Ecol. 2007, 16, 3759-3767. 
18. Ellis, J.R.; Burke, J.M. EST-SSRs as a resource for population genetic analyses. Heredity 2007, 99, 125-132.

19. Wang, X.W.; Luan, J.B.; Li, J.M.; Bao, Y.Y.; Zhang, C.X.; Liu, S.S. De novo characterization of a whitefly transcriptome and analysis of its gene expression during development. BMC Genomics 2010, 11, 400 .

20. You, F.M.; Huo, N.; Gu, Y.Q.; Luo, M.C.; Ma, Y.; Hane, D.; Lazo, G.R.; Dvorak, J.; Anderson, O.D. BatchPrimer3: A high throughput web application for PCR and sequencing primer design. BMC Bioinforma. 2008, 9, 253.

21. National Center for Biotechnology Information, U.S. National Library of Medicine. Available online: http://www.ncbi.nlm.nih.gov/tools/primer-blast/index.cgi?LINK_LOC=BlastHome (accessed on 30 July 2012).

22. Yeh, F.C.; Boyle, T.J.B. Population genetic analysis of co-dominant and dominant markers and quantitative traits. Belg. J. Bot. 1997, 129, 157.

23. Botstein, D.; White, R.L.; Skolnick, M.; Davis, R.W. Construction of a genetic linkage map in man using restriction fragment length polymorphisms. Am. J. Hum. Genet. 1980, 32, 314-331.

24. Raymond, M.; Rousset, F. GENEPOP (version 1.2): Population genetic software for exact tests and ecumenicism. J. Hered. 1995, 86, 248-249.

25. Rice, W.R. Analyzing tables of statistical tests. Evolution 1989, 43, 223-225.

26. Van Oosterhout, C.; Hutchinson, W.F.; Wills, D.P.M.; Shipley, P. MICRO-CHECKER: Software for identifying and correcting genotyping errors in microsatellite data. Mol. Ecol. Notes 2004, $4,535-538$.

(C) 2012 by the authors; licensee MDPI, Basel, Switzerland. This article is an open access article distributed under the terms and conditions of the Creative Commons Attribution license (http://creativecommons.org/licenses/by/3.0/). 